\title{
On Design Methods for Sampled-Data Systems
}

\author{
Geir Dullerud and John Doyle*
}

\begin{abstract}
In this paper we compare, via example, the standard approaches to sampled-data design with recently developed direct design methods for these hybrid systems. Simple intuitive examples are used to show that traditional design heuristics provide no performance guarantees whatsoever. Even when the sampling rate is a design parameter that can be chosen as fast as desired, using design heuristics can lead to either severe performance degradation or extreme over-design. These effects are apparently well-known to practitioners, but may not be widely appreciated by the control community at large. The paper contains no new theoretical results and is intended to be of a tutorial nature.
\end{abstract}

\section{Introduction}

Sampled-data systems are hybrid systems consisting of interacting discrete-time and continuous-time subsystems, for example when digital hardware is used to implement a controller for a continuous-time process. Sampled-data systems also arise because some systems only permit discrete measurements; notably, for many chemical and biomedical systems the only sensing instruments available produce discrete signals.

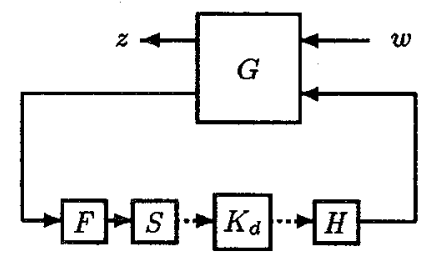

Figure 1: General Sampled-data Configuration

Figure 1 shows a widely used model for sampled-data systems: $G$ is a continuous-time plant, which is to be controlled by the discrete-time controller $K_{d}$; the $S$ and $H$ blocks in the diagram are sample and hold devices, respectively, and $F$ is a low-pass data filter. The objective of the controller is to attenuate the gain from the input $w$ to the oupat $z$. The sampled-data design problem is therefore:

Given a continuous-time plant $G$, find a discrete-

time compensator $K_{d}$ that achieves the performance requirements.

Sampled-data systems have been studied since the $50 \mathrm{~s}$ (see, e.g. [11]), and there are three basic approaches to the above design problem. These are illustrated in Figure 2. The traditional approaches to sampled-data design follow the paths on the perimeter of Figure 2: one of these approaches is to design a continuous-time controller $K$ as the first step; then to discretize $K$ using a heuristic to arrive at the final compensator $K_{d}$. The second approach is to discretize the plant $G$, and then to perform a discrete-time design on the plant $G_{d}$ to obtain $K_{d}$. The former method ignores implementation during the design stage, and the latter technique of dis-

"the authors are with the Department of Electrical Engineering, California Institute of Technology, Pasadena, CA 91125

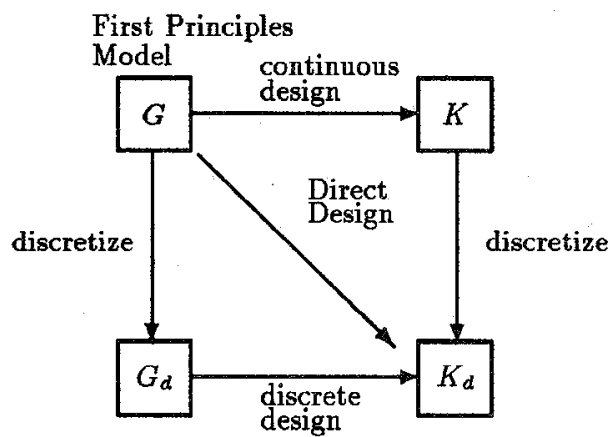

Figure 2: Design Methods

cretizing the plant does not account for intersample behavior during the design stage. The above approaches are described in the two standard texts [3] and [8], and rely on the sampling rate being significantly faster than the dynamics to be regvlated. These methods have considerable practical merit in many situations, but because they are inexact there has been considerable research activity recently (see references for a partial list) into direct sampled-data design. This is shown by the diagonal line in Figure 2. In direct sampled-data design the compensator $K_{d}$ is obtained taking into account both implementation considerations and intersample behavior.

Sampled-data design problems can be classified into two broad categories:

- the sampling rate is a fixed parameter

- the sampling rate is a design variable

In the first of these, the designer is given a fixed sampling rate for the compensator, and must produce a design that operates at this rate. The advantages of using direct sampled-data design are clear in this scenario when the sampling rate specified does not satisfy engineering rules-of-thumb or heuristics. However, when the sampling rate can be chosen by the control engineer the benefits of direct design are not (at first) so apparent.

In the next section we present two simple examples that clearly illustrate these points. A continuous-time design example demonstrates that even at high sample rates, standard design heuristics provide no performance guarantees. A discrete-time example shows that considering the open-loop bandwidth of a system can lead to extreme over-sampling in the controller implementation. The examples used are intentionally very simple and naive, so that the problems in the heuristic designs are easily recognized and understood. This also means that they are readily correctable by recognizing special stracture in the problem and hand-crafting a solution. This task becomes complex, and perhaps impossible, in large multivariable designs. Direct design methods account for both implementation and intersample behavior in an automated procedure. While this may be unnecessary in many situations, it is convenient to have true sampled-data methods available, thus freeing the the control engineer to focus her engineering judgement on tasks where it is necessarily required.

\section{Examples}

To construct the examples we use the loop-shaping arrangement of Figure 3, where performance is measured by the $L_{2}$ induced norm of $w \mapsto z$. When $T$ and its left-inverse 
$T^{-1}$ are both in $H_{\infty}$, the optimal controller $K_{\text {opt }}$ for this setup is $-T^{-1}$, with performance $\gamma_{o p t}=\sqrt{2}$. In the following two subsections we use this configuration, varying $T$, and perform sampled-data designs using all three approaches outlined above.

\subsection{Continuous-time Design}

Suppose we have a controller $K$ for the configuration of Figure 3 and want to discretize it for implementation. There are numerous methods to accomplish this and perhaps the best is using the Tustin transformation: we use

$$
s \mapsto \frac{2}{h} \frac{z-1}{z+1}
$$

to convert $K(s)$ to $K_{d}(z)$, where $h$ is the sampling period. Most books suggest that this discretization be carried out with a sampling rate of 20-100 times the closed loop bandwidth. The goal of all these discretization methods is to ensure that

$$
K(j \omega / h) \approx K_{d}\left(e^{j \omega}\right)
$$

when $\omega / h$ is in the bandwidth of the closed loop.

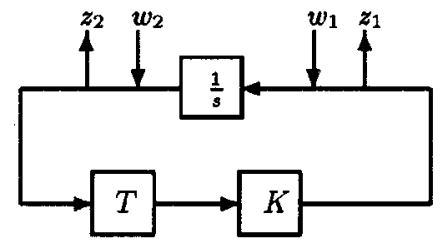

Figure 3: Loop-shaping Configuration

Concentrating on the configuration in Figure 3, suppose that we perform a sampled-data implementation of the optimal controller as in Figure 1 with

$$
T(s)=\frac{s^{2}+3 \cdot 10^{-4} s+0.4}{(s+1)^{2}} .
$$

Then we discretize $K_{\text {opt }}(s)=-T^{-1}(s)$ using (1). Noting the closed loop bandwidth is $1 \mathrm{rad} / \mathrm{s}$, we adhere to the standard design heuristics and select a sampling rate of $100 \mathrm{rad} / \mathrm{s}$ and $F(s)=\frac{1}{0.1 s+1}$. Checking the $L_{2}$ induced norm of this implementation we get

$$
\|w \mapsto z\| \approx 200 .
$$

This is easily seen graphically by discretizing the entire closed loop and plotting the discrete-time fourier transform of the system. The plot has a large narrow spike at a single frequency where even a small mismatch in (2) is critical. If instead the heuristic $K_{d}=S K H$ is applied, the system is closed-loop unstable.

However, carrying out a direct sampled-data design on the configuration in Figure 3 with $F$ as above, we get

$$
\gamma_{s d_{o p t}}:=\|w \mapsto z\| \approx 2.02,
$$

which is near the continuous-time optimum of $\sqrt{2}$.

It is possible to make this discrepancy much larger by moving the zeros of $T$ closer to the imaginary axis. The above discretization problem can be avoided by choosing a modified Tustin transformation:

$$
s \mapsto \frac{q}{h} \frac{z-1}{z+1},
$$

where $q$ is a warping parameter which is adjusted to make (2) match at the critical frequency. However, the dynamics of $T$ can be made more complicated so that there are two critical frequency points, and it is impossible match both pointo even with the modified Tustin transformation. A higher order mapping from the half-plane to the disc would be required.

\subsection{Discrete Design}

Again considering Figure 3, let

$$
T=\left[\begin{array}{l}
1+R \\
1-R
\end{array}\right]
$$

where $R$ is some stable system. Then the optimal controller is

$$
K_{\text {opt }}=-\frac{1}{2}\left[\begin{array}{ll}
1 & 1
\end{array}\right]
$$

regardless of $R$. If $R$ has a very large bandwidth, then discretizing the design by considering the open loop plant bandwidth would lead to an unnecessarily high sampling rate; in fact $R$ could be such that the bandwidth of the map from $w_{1}$ and $w_{2}$ to the controller input would be arbitrarily large. In contrast, regardless of $R$, performing the sampled-data implementation of Figure 1 by discretizing $K_{\text {opt }}$, with $20 \mathrm{rad} / \mathrm{s} \mathrm{sam-}$ pling rate and $F(s)=\frac{1}{0.5 s+1}$ yields $\|w \mapsto z\|_{L_{2} \rightarrow L_{2}}=2.35$. A direct sampled-data design, at the same rate, gives a controller with performance $\gamma_{s d_{o p t}} \approx 1.65$.

\section{References}

[1] M. Araki and Y. Ito, "Frequency-response of sampled-data systems I: open-loop considerations," Proc. of IFAC 12th World Congress.

[2] M. Araki and $Y$. Ito, "Frequency-response of sampled-data systems II: closed-loop considerations," Proc. of IFAC 12th World Congress.

[3] K.J. Astrom and B. Wittenmark, Computer Controlled Systems, Prentice-Hall, Englewood Cliffs, 1984.

[4] B. Bamieh, and J.B. Pearson, "A General Framework for Linear Periodic Systems with Application to $H_{\infty}$ Sampled-data Control," IEEE Trans. Auto. Control, vol. 37, pp. 418-435, 1992.

[5] B. Bamieh, J.B. Pearson, B.A. Francis, and A. Tannenbaum, "A Lifting Technique for Linear Periodic Systems with Applications to Sampled-data Control," Systems and Control Letters, vol. 8, 1991.

[6] T. Chen and B.A. Francis, "Stability of sampled-data feedback systems," IEEE Trans. Auto. Control, vol. 36, pp. 50-58, 1991.

[7] G.E. Dullerud and K. Glover, "Robust Stabilization of Sampled-data Systems to Structured LTI Perturbations," IEEE Trans. Auto. Control, vol. 38, pp. 1497-1508, 1993.

[8] G.F. Franklin, J.D. Powell, and M.L. Workman, Digital Control of Dynamic Systems, Addison Wesley, Reading, 1990.

[9] S. Hara, and P.T. Kabamba, "Worst case analysis and design of sampled-data control systems," Proc. CDC, 1990.

[10] S. Hara, M. Nakajima, and P.T. Kabamba, "Robust Stabilization in Digital Control Systems," Proc. MTNS, 1991.

[11] R.E. Kalman, and J.E. Bertram, "A Unified Approach to the Theory of Sampling," J. of Franklin Institute, vol. 267, no. 5, 1957.

[12] N. Sivashankar and P.P. Khargonekar, "Robust Stability and Performance Analysis of Sampled-Data Systems," IEEE Trans. Auto. Control, vol. 38, pp. 58-69, 1993.

[13] W. Sun, K.M. Nagpal, and P.P. Khargonekar, " $H_{\infty}$ Control and Filtering for Sampled-data Systems," IEEE Trans. Auto. Control, vol. 38, pp. 1162-1175, 1993.

[14] H.T. Toivonen, "Sampled-data control of continuous-time systems with an $H_{\infty}$ optimality criterion," Rep. 90-1, Dept. Chemical Eng., Abo Akademi, Finland, Jan. 1990.

[15] P.M. Thompson, R.L. Daily and J.C. Doyle, "New conic sectors for sampled-data system feedback systems," Systems and Control Letters, vol. 7, pp. 395-404, 1986.

[16] P.M. Thompson, G. Stein and M. Athans, "Conic Sectors for Sampled-Data Feedback Systems", Systems and Control Letters, vol. 3, pp. 77-82, 1983.

[17] Y. Yamamoto, "A new approach to sampled-data control systems - A function space viewpoint with applications to tracking problems," CDC Proceedings, pp. 1882-1887, 1990. 